\title{
Estudos etnoictiológicos sobre o pirarucu Arapaima gigas na Amazônia Central
}

\author{
Liane GALVÃO DE LIMA', Vandick da Silva BATISTA²
}

\begin{abstract}
RESUMO
O presente estudo visou identificar saberes comuns entre o conhecimento científico e o conhecimento local sobre a ecologia e biologia do pirarucu (Arapaima gigas), contribuindo com informaçôes úteis para a implementação e consolidação de projetos de manejo participativo pesqueiro na regiáo. Foram realizadas 57 entrevistas semi-estruturadas, com pescadores profissionais de Manaus e pescadores de subsistência de Manacapuru durante o período de junho a dezembro do ano de 2002. Foi observado que os pescadores profissionais possuem informaçóes igualmente precisas e abrangentes em relação aos saberes dos pescadores ribeirinhos de subsistência nos aspectos de reprodução, predação, migração, crescimento e mortalidade. Os aspectos que náo são equivalentes entre os pescadores profissionais comerciais citadinos e ribeirinhos de subsistência são nos aspectos de tipo de alimentação e no tamanho de recrutamento pesqueiro. Concluímos que os pescadores da Amazônia central possuem os conhecimentos necessários que possibilitam o manejo participativo do pirarucu, como um profundo saber nos aspectos comportamentais, biológicos e ecológicos desta espécie, podendo assim contribuir de fato com a participação de gestão nos recursos pesqueiros locais.
\end{abstract}

PALAVRAS-CHAVE: Etnobiologia, pesca artesanal, pesca profissional, manejo participativo.

\section{Ethnoictiology studies on Pirarucu (Arapaima mock-ups) in Central Amazon}

\begin{abstract}
Present study it aimed at to identify to know common between scientific knowledge and local knowledge on ecology and biology of pirarucu (Arapaima mock-ups), contributing with useful information for implementation and consolidation of projects of participative handling fishing boat in region. 57 half-structuralized interviews had been carried through, with fishing of Manaus and Manacapuru during period of June to December of year 2002. It was observed that professional fishermen also have accurate and comprehensive information in relation to knowledge of subsistence fishermen in coastal aspects of reproduction, predation, migration, growth and mortality. Aspects that are not equivalent among commercial fishermen and coastal urban dwellers are subsistence aspects of type of power and size of fishing recruitment. We conclude that fishermen of central Amazonia have necessary knowledge that allow participatory management of bass, and a deep knowledge in behavioral, biological and ecological processes of this kind, so you can actually contribute to the participation of management in local fisheries.
\end{abstract}

KEYWORDS: Ethnobiology, fishing, commercial fishing, participatory management.

\footnotetext{
1 Universidade Federal do Amazonas. UFAM. - Av. Gen. Rodrigo Otávio Jordão Ramos. 3000. Mini-Campus. Bairro Coroado I. 69077-000. Manaus, AM - Brasil. Telefone: (092) 3307-6411. E-mail: liagalva0@gmail.com

2 Universidade Federal de Alagoas, Instituto de Ciências Biológicas e da Saúde, Núcleo de Ecologia e Biodiversidade. UFAL/ICBS - Praça Afrânio Jorge s/n. Prado. 57010-020. Maceió, AL - Brasil. Telefone: (82) 32235613 Fax: (82) 32212501. E-mail: vbatista@pesquisador.cnpq.br
} 


\section{INTRODUÇÃO}

Etnoictiologia é o modo como o conhecimento, os usos e os significados dos peixes ocorrem nas diferentes sociedades humanas, a qual foi definida como a busca da compreensáo do fenômeno da interação da espécie humana com os recursos ícticos, englobando-se aspectos tanto cognitivos quanto comportamentais (Marques 1995). Costa-Neto (2001) definiu como o estudo científico das relaçóes entre homem com os peixes.

Ela inclui-se na etnozoologia (estudo científico das relaçóes homens e animais), que por sua vez faz parte de uma disciplina mais abrangente, a etnobiologia (Costa-Neto 2001).

Vários estudos tem analisado e interpretado as informaçôes etnoecológicas adquiridas junto aos pescadores com base na ecologia (Ruddle 2001), gerando informaçóes condizentes com a literatura de ictiologia.

No Brasil pesquisas etnoictiológicas tem sido realizadas com enfoque na etnotaxonomia (classificação e nomenclatura) de peixes por pescadores de várias regióes, com a Costa Sudeste, Nordeste e um rio Amazônico (Begossi e Garavello 1990). Resultados de estudos etnoictiológicos demonstram que os pescadores exibem um conhecimento detalhado sobre o comportamento dos peixes, o qual é aplicado nas estratégias de pesca e geralmente condiz com as observaçóes científicas (Marques 1991,1995; Costa-Neto 2001).

Apesar do reconhecimento da importância das populaçóes pesqueiras artesanais e, da necessidade do melhor conhecimento da dinâmica de suas pescarias, pouca informação etnoictiológica sobre o assunto tem sido levantada na nossa região que tem como a pesca uma das atividades mais tradicionais, desempenhando um papel importante na economia e no processo de ocupação humana na regiáo (Santos e Ferreira 1999). Representa também um importante elemento da cultura regional (Furtado 1993).

O conhecimento dessas interaçóes homem-ambiente pode subsidiar na formulaçáo de programas de manejo e de estratégias de co-manejo (Seixas e Begossi 2001). Além disso, é fundamental ressaltar a importância do planejamento participativo, que envolve a comunidade em duas etapas, desde a fase das coletas de dados, passando pela formulação do programa de gestão até a sua implantação e monitoramento (Clark 1996).

Portanto, a atividade pesqueira artesanal, requer todo um conhecimento etnoecológico que possibilite à exploração sustentável do recurso pesqueiro. Já afirmava Marques (1995), que os pescadores detêm o saber e o saber fazer relacionado com a estrutura e a função do ecossistema a que estão vinculados.

O pirarucu Arapaima gigas, peixe endêmico da bacia amazônica habita principalmente, lagos de várzeas e florestas inundadas (Castello 2008). Possui respiração aérea obrigatória e pode atingir $200 \mathrm{~kg}$ de massa corpórea e $3 \mathrm{~m}$ de comprimento (Castello 2004). É a espécie de peixe mais consumida e comercializada, iguaria tradicional da culinária amazônica urbana e ambicionado recurso pesqueiro (Murrieta 2001). É considerado o "bacalhau brasileiro", devido ao excelente sabor de sua carne, particularmente quando beneficiada seca e salgada (Fontenele e Vasconcelos 1982; Imbiriba 2001). O que determina o seu alto valor consiste no seu grande porte, tornando-se então, um alimento rico em proteína, superando a carne do salmão, sardinha e carne bovina, submetidas o igual tratamento (Neves 2000). No entanto, dados de Petrere Jr. (1992), Crossa e Petrere (1999) já indicavam que a densidade de espécie grande, tem diminuído próxima de cidades, devido a pressão da pesca nos últimos anos. Ressalta-se que toda a pesca do pirarucu foi proibida no início de 2001, mas as pescas ilegais continua até hoje. No entanto, desde 1999, a exploração limitada do Arapaima gigas era permitido dentro da Reserva Biológica Sustentável de Mamirauá, e posteriormente foi estendida para algumas regióes do Estado por um período sustentável ao estoque do pirarucu (Hrbek et. al. 2007).

Nesse contexto, o presente estudo pretendeu avaliar aspectos etnoictiológicos dos pescadores profissionais e ribeirinhos da Amazônia Central sobre o pirarucu, a fim de verificar se os pescadores comerciais possuem informaçóes igualmente precisas e abrangentes em relação aos saberes locais dos pescadores de subsistência sobre a dinâmica de população do pirarucu e, como também, comparar o saber etnoictiológico de pescadores citadinos comerciais e de ribeirinhos de subsistência em relação ao saber científico já acumulado, permitindo sua utilização no manejo participativo desta espécie.

\section{MATERIAL E MÉTODOS}

A coleta de dados primários foi efetuada na balsa de desembarque de pescado localizada na Frente da Feira da Manaus Moderna, Centro. E uma zona rural do município de Manacapuru (AM) Incluindo toda Costa do Pesqueiro, Costa do Catalão, Lago do Cururu e Lago do Jacaré, entre as coordenadas geográficas: $61^{\circ} 00$ a $60^{\circ} 45^{\prime} \mathrm{S}$ a $3^{\circ} 30^{\prime}$ a $3^{\circ} 40^{\prime} \mathrm{W}$.

Os dados secundários sobre a biologia e ecologia da espécie em estudo foram obtidos por meio de revisóes bibliográficas durante todo o período de estudo (2001-2003). Estas fontes bibliográficas incluíram teses, monografias, artigos científicos e livros especializados no assunto.

$\mathrm{O}$ questionário semi-estruturado apresentava perguntas chaves e também questóes abertas, as quais incluíram 45 tópicos, abordando primeiramente os aspectos pessoais, experiências e relaçóes sociais na pesca e, posteriormente os aspectos biológicos e ecológicos (reprodução, alimentação, 
predação, crescimento, migração, mortalidade e recrutamento) da espécie em foco.

Os entrevistados foram selecionados aleatoriamente. Por se tratar de um estudo de etnoconhecimento, as expressóes e palavras nativas foram mantidas a fim de garantir maior confiança entre as partes.

As entrevistas foram realizadas de modo individual, durante o período de julho a dezembro do ano de 2002, tiveram duração de 40 minutos a cerca de 2 horas. Foram todas registradas por escrito, e logo em seguida inseridas num banco de dados, onde foram posteriormente efetuadas as consultas e análises.

Foi realizada uma análise comparativa dos dados científicos com o conhecimento local e também entre os pescadores. Para isto foram utilizados tabelas dinâmicas, gráficos de setores, histogramas, médias e intervalos de confiança. O teste estatístico $Z$ foi utilizado na comparação de proporções em relação à freqüência de informaçóes (Levine et al. 2000). O pressuposto da normalidade foi avaliado por KolmogorovSmirnov e da homocedasticidade por Bartlett (Zar 1999), como também para analisar as diferenças entre as proporçóes de amostras obtidas nas respostas dos pescadores citadinos, aquele que vive em cidades e já perdeu sua ligaçáo com a terra (Furtado 1993) e pescadores ribeirinhos, são os pescadores interioranos que vivem na zona rural e tem alguma relação com a terra, onde a pesca é renda parcial de suas atividades, podendo ser principal ou complementar de outras atividades relacionadas ao campo (Furtado, 1993).

\section{RESULTADOS E DISCUSSÃO}

Foram realizadas 57 entrevistas semi-estruturadas no total, destas 31 entrevistas com os pescadores profissionais citadinos de Manaus, e 26 entrevistas com os pescadores ribeirinhos comerciais e de subsistência das áreas rurais de Manacapuru.

\section{Conhecimentos relacionados com o comportamento reprodutivo}

Não foi observada diferença significativa $(p>0,01)$ entre as informaçóes dadas pelos pescadores citadinos e ribeirinhos em relação ao comportamento reprodutivo do pirarucu quanto aos aspectos de tamanho e idade de primeira maturação e tamanho em que o peixe atinge a maturidade completa.

Os pescadores da Amazônia Central relataram que o peixe começa reproduzir com $159 \pm 43 \mathrm{~cm}$ de tamanho e $4 \pm 2$ anos de idade. E atinge a maturidade completa com a média de $257 \pm 72 \mathrm{~cm}$ de tamanho. Informaram ainda que a espécie:

1. reproduz no período da enchente;

2. apresenta o costume de desovar em buracos no fundo do lago "faz panela", e descreveram os cuidados parentais com suas crias (Figura 1);
3. desova uma vez por ano;

4. tem fecundidade classificada entre 0-500 ovos por desova.

Para a idade de maturação plena foi verificada diferença significativa $(\mathrm{p}<0,01)$, onde os pescadores citadinos indicaram que o pirarucu atinge a plena maturação com $8 \pm 1,6$ anos de idade e os ribeirinhos registraram $6 \pm 2,1$ anos de idade para maturação completa do pirarucu.

Esses dados demonstram equivalências com as bibliografias científicas, onde, Lowe-McConnell (1975) registrou 212 $\mathrm{cm}$ para tamanho de primeira maturação do A. gigas. Em Mamirauá segundo Queiroz (2000) o pirarucu começa a reproduzir com $165-168 \mathrm{~cm}$ por volta dos 5 anos. Fontenelle (1948), Imbiriba (1994) estudaram o pirarucu em cativeiro, e registraram que sua reproduçáo ocorre com o peso de 40-50 $\mathrm{kg}$, a partir do $5^{\circ}$ ano quando já apresenta mais de $160 \mathrm{~cm}$ de comprimento total. Neves (2000) após a análise de estágios de maturidade das gônadas do pirarucu no rio Pacaya e Samiria no Peru concluiu que esta espécie começa amadurecer quando atinge o peso de 40-50 kg entre $160-185 \mathrm{~cm}$ após o $4^{\circ} \mathrm{e}$ $5^{\circ}$ ano de vida determinado através de exames das vértebras e pela curva de crescimento. Para a espécie em cativeiro na Amazônia peruana, Guerra (1980) determinou a primeira maturaçáo com $185 \mathrm{~cm}$ e com a idade de 5 anos.

Comparando estas informaçôes com o conhecimento científico vimos que existe coerência, o pirarucu do rio Pacaya e Samiria no Peru (Guerra 1980), desova durante o ano todo com um período de máxima intensidade de agosto a dezembro, sendo que o pico mais notável é no mês de novembro e, de mínima intensidade, é entre março e maio. Neves (2000) sugere que o pirarucu que apesar de haver várias desovas durante um período de reprodução, pois os óvulos amadurecem sucessivamente, a desova pode não ser anual, mas sim ocorrendo a cada dois anos, o que reduz a fecundidade total. Este resultado assemelha-se com os resultados descritos por Queiroz (2000).

De acordo com Fontenele (1948) o pirarucu em cativeiro, quando vai reproduzir, dá preferência a locais sem vegetação aquática flutuante, embora depois da desova, os reprodutores conduzissem os alevinos para locais obrigados ou não de
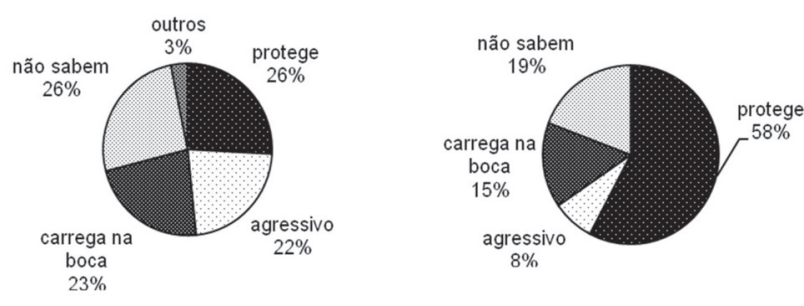

Figura 1 - Tipos de cuidados parentais do pirarucu segundo os pescadores citadinos (esquerda; $n=30$ ) e ribeirinhos (direita; $n=26$ ) da Amazônia Central. 
vegetação aquática. A profundidade da água varia entre os limites 0.80 a $1,00 \mathrm{~m}$.

Bard e Imbiriba (1986), Ferreira et al (1998) descreveram que os ninhos do pirarucu são cavados no fundo dos lagos com a cooperaçáo de ambos os pais em pouca água corrente.

A Tabela 1 mostra de forma resumida uma comparaçáo do ciclo reprodutivo do pirarucu entre as dados citados pelos pescadores da Amazônia Central e os encontrados na literatura científica.

\section{Conhecimentos relacionados com ecologia trófica}

Segundo os pescadores da Amazônia Central, o pirarucu tem uma alimentaçáo diversificada registrando como sua preferência alimentar, o peixe e o camarão. Indicaram o lago durante os períodos de enchente e vazante. Vale ressaltar, que não há mudanças nos itens da dieta alimentar nas épocas de subida e descida das águas sendo que alguns pescadores enfatizaram que diminui a oferta de alimentos durante o período da seca, não foi observado diferenças significativas ( $p$ > $0,01)$ entre as respostas dos pescadores citadinos e ribeirinhos (Figura 2).

Essas informaçóes apresentam-se equivalentes com os encontrados na literatura científica, onde observamos que Queiroz (2000) examinou o conteúdo estomacal de 232 pirarucus de ambos os sexos de vários tamanhos originados de uma Coleção Referencial de Mamirauá de tratos digestivos do pirarucu conservados em uma solução de álcool (30-40\%) e formol (15\%), referentes ao período de abril de 1993 e junho de 1995, descrevendo que o pirarucu alimenta-se tanto de vertebrados como invertebrados. Dos vertebrados, os que se destacaram foram espécies de outros peixes, exemplos: "tamoatá" (Mecalechy thoracatam), "bodó" (Hypostomus spp., Loricariichthys spp. e Gyptarichthys gibbcepes), "mandi" (Pimelodus spp. e Pimelodella cristalta), "traíra" (Hoplias malabaricus), "acarâ" (Apistogramma spp., Heros appendiculatus e Mesonauta insignis), "rabeca" (Megalodoras uranoscafus), "sarapó" (Sternopygidae, Gymnotidae, Rhamphichthyidae,

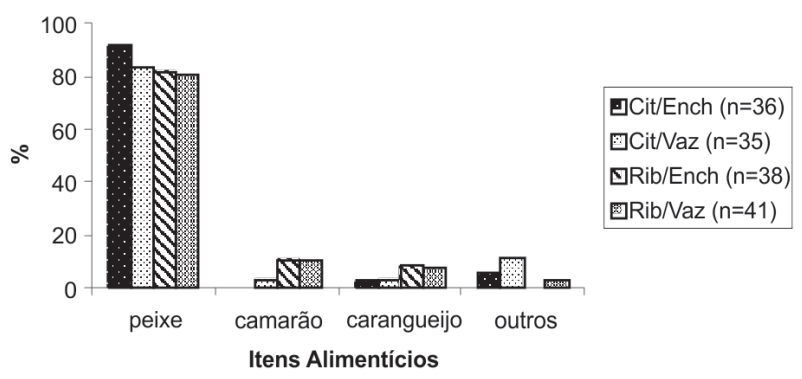

Figura 2 - Alimentação do pirarucu segundo os pescadores citadinos (Cit) e ribeirinhos (Rib) da Amazônia Central durante o período de enchente (Ench) e vazante (Vaz).
Tabela 1 - Comparação entre as informações dos pescadores da Amazônia central e da literatura sobre o comportamento reprodutivo do pirarucu

CITAÇÕES DAS

ENTREVISTAS COM OS PESCADORES

\begin{tabular}{|c|c|}
\hline $\begin{array}{l}\text { "0 peixe começa produzir } \\
\text { com } 150 \mathrm{~cm} \text { e } 4 \text { anos de } \\
\text { idade" }\end{array}$ & $\begin{array}{l}\mathrm{L}_{50}: 212 \mathrm{~cm} \text { e } 6 \text { anos (Allsopp, 1958; } \\
\text { Lübling, 1964; Lowe-MacConnel, 1975); } \\
160 \mathrm{~cm} \text { e } 5 \text { anos (Fontenelle, 1948; } \\
\text { Imbiriba, 1994). }\end{array}$ \\
\hline $0 \mathrm{~cm}$ com 8 anos de & $\mathrm{L}_{100}: 311,7 \mathrm{~cm}$ e 10 anos (Queiroz, 2000) \\
\hline
\end{tabular}
idade"

Durante 0 ano todo - pico em nov-dez "O pirarucu produz filhote (enchente) (Lüling, 1964; Flores, 1980 apud na enchente" Neves, 2000; Queiroz, 2000; Lübling, 1964; Lowe-McConnel, 1975; Alssopp, 1958)

Florestas inundadas, constroem ninhos no fundo do lago (Sawaya, 1946; Alssopp,

"0 pirarucu desova no buraco no fundo do lago, faz panela" 1958; Lübling, 1964; Lowe-McConnel, 1975; Bard \& Imbiriba, 1986; Ferreira et al., 1998; Queiroz, 2000.)

"0 macho protege 0 filhote, fica agressivo"

Protegem a prole, principalmente os alevinos recém nascidos (Neves, 2000); Cuidado com o ninho, principalmente executado pelo macho (Queiroz, 2000).

"O pirarucu reproduz uma vez por ano"

Parcelada (Lübling, 1964; Lowe-McConnel, 1975; Alssopp, 1958; Neves, 2000; Queiroz, 2000)

"O peixe tem muitos ovos 11000 ovócitos (Bard \& Imbiriba, 1986); (0-500 ovos)" 20327 ovócitos (Queiroz, 2000).

Hypopomidae, Apteronotidae e Eigenmanniidae), "Chorona" (Curimatella sp.), "peixe-cachorro" (Acestrorhynchus e Rhaphiodon), "cascudinha" (Psectrogaster rutilordes e $P$. amazonica), "jeju" (Hoplerythrinus unitaeniatus) e "branquinha"(Potamorhina altamazonica).

Eventualmente alimenta-se de vegetais (pequenos brotos, algumas sementes e flores, fragmentos de macrófitas aquáticas Eichhornia sp.). Em pirarucus jovens, além de peixes, foram encontrados invertebrados como insetos (Coleoptera, Diptera, Odonata e Hemiptera.), crustáceos e moluscos. Os de maiores importância foram os macrocrustáceos décapodes (Malacostraca), isto é caranguejo (Sylviocarcinus pictus e Dilocarcinus pagei) e camarão (Macrobrachium amazonicum). Dos microcrustáceos foram encontrados Ostracoda e Conchostraca (Branchiopoda), presentes somente nos estômagos de animais muitos jovens (menos que $50 \mathrm{~cm}$ CT). Oliveira et al (2000) em estudo da espécie em ambiente natural no Mato Grosso, verificou que os peixes jovens tem preferências alimentares por insetos, microcrustáceos e gastrópodes, 
Os principais predadores do pirarucu conhecidos pelos pescadores da Amazônia Central são outros tipos de peixes, que se alimentam dos seus filhotes, e o jacaré (Melanosuchus Niger) durante toda sazonalidade das águas, não observando diferenças significas $(p>0,01)$ entre os tipos de pescadores citadinos e ribeirinhos.

Corroborando com Neves (2000), a qual descreve que o pirarucu jovem tem como "predadores principais" as aves (Anhinga anhinga, Ceryle torquata, Phalacrocorax brasilianus) que os atacam para comê-los. Outros predadores importantes do pirarucu jovem são: a piranha (Serrassamus spp.), e o jeju (Hopterythrinus sp.). Os ocasionais são Cichla monoculus (tucunaré) e Astronotus ocellatus (Acará açu). Mikdalski (1957) registrou que o jacaré (Caiman yacare) é um outro predador em potencial de pirarucus jovens de $40-50 \mathrm{~cm}$.

\section{Conhecimentos relacionados com comportamento migratório}

Em relaçáo ao comportamento migratório do pirarucu, os pescadores informaram que o peixe migra somente no período da cheia e enchente de um lago para outro lago para desovar. $\mathrm{E}$ durante os períodos de vazante e seca o peixe náo migra, não havendo diferenças significativas $(p>0,01)$ entre os dados de pescadores citadinos e ribeirinhos.

De modo geral, os pescadores percebem que esta espécie é considerada sedentária conforme já descritos nas literaturas especializadas (Queiroz 2000).

\section{Conhecimentos relacionados com crescimento}

Quanto ao crescimento do pirarucu, os pescadores registraram a média de tamanho do pirarucu com a idade de 1 ano é $32+/-18,6 \mathrm{~cm}$ de comprimento. Com a idade de 2 anos, é $66,6+/-33,5 \mathrm{~cm}$ de comprimento. Com 3 anos, indicaram que o peixe pode alcançar a média de tamanho de $73+/-24,2 \mathrm{~cm}$ e com 4 anos, a média de tamanho é de 190 $+/-155 \mathrm{~cm}$ náo havendo diferenças significativas entre os pescadores $(\mathrm{p}>0,01)$.

Comparando essas informaçóes com os da literatura científica encontramos razoável equivalência com resultados de taxa de crescimento obtidos por Queiroz (2000), utilizando dados de capturas no período de 1993-1998 (Tabela 02).

Tabela 02 - Crescimento do pirarucu segundo os pescadores da Amazônia Central comparado com a literatura científica

\begin{tabular}{cccc}
\hline Idade & Pescadores & Literatura & Autor \\
\hline 1 & $32 \mathrm{~cm}$ & $55-56 \mathrm{~cm}$ & \\
2 & $66,6 \mathrm{~cm}$ & $73-75 \mathrm{~cm}$ & \\
3 & $73 \mathrm{~cm}$ & $91-94 \mathrm{~cm}$ & Queiroz, 2000 \\
4 & $190 \mathrm{~cm}$ & $106-107 \mathrm{~cm}$ & \\
\hline
\end{tabular}

\section{Conhecimento relacionado com a mortalidade e recrutamento pesqueiro}

Para os pescadores da Amazônia Central o que mais mata pirarucu na fase larval e juvenil é a mortalidade por causas naturais, neste caso eles apontaram a predação. E na fase adulta os pescadores indicaram a pesca como a principal causa de mortalidade destes peixes, onde não foram registradas diferenças significativas $(p>0,01)$ entre as respostas dos pescadores citadinos e ribeirinhos (Figura 3).

Fato este, que podemos confirmar na literatura sobre o aumento do esforço pesqueiro os quais esta espécie tem sofrido nos últimos anos. Souza e Val (1991) registraram que dados da ex-SUDEPE mostraram, que no estado do Amazonas, as capturas desta espécie caíram de 1140 ton. em 1979 para 364 ton. em 1986. Na Amazônia Central, Batista (1998) registrou a produção pesqueira anual para a espécie 67,13 ton. em 1994, e 15,80 ton. em 1995. De acordo com Isaac e Ruffino (2000), foi desembarcado nos anos de 1992 a 1996 uma média de 18,5 toneladas por ano em Santarém. Imbiriba (2001) relata que na Amazônia o pirarucu é capturado pela pesca comercial com peso variando entre $30 \mathrm{a} 40 \mathrm{~kg}$.

Em relação ao tamanho de captura do pirarucu foram verificadas diferenças significativas ( $\mathrm{p}<0,01)$ entre os tamanhos apontados pelos pescadores, os citadinos $(\mathrm{n}=25)$ registraram um tamanho médio de $151 \pm 67 \mathrm{~cm}$. Os pescadores ribeirinhos $(\mathrm{n}=21)$ afirmaram que o pirarucu começa a ser capturado na média dos $51 \pm 55 \mathrm{~cm}$ de comprimento.

Os dados citados pelos pescadores ribeirinhos são equivalentes aos citados por Junk e Honda (1976) em Manaus, na década de 70 , grande parte dos animais desembarcados possuía comprimento total inferior a $100 \mathrm{~cm}$; e com Neves (2000), registrando que de 810 pirarucus amostrados em 1996, em Santarém, mais de $80 \%$ estavam abaixo de 150 $\mathrm{cm}$. Já os dados registrados pelos pescadores ribeirinhos são congruentes aos resultados encontrados por Queiroz (2000) onde este afirma que a captura do pirarucu em Mamirauá esta sendo efetuado a partir de $149-151 \mathrm{~cm}$ comprimento total.

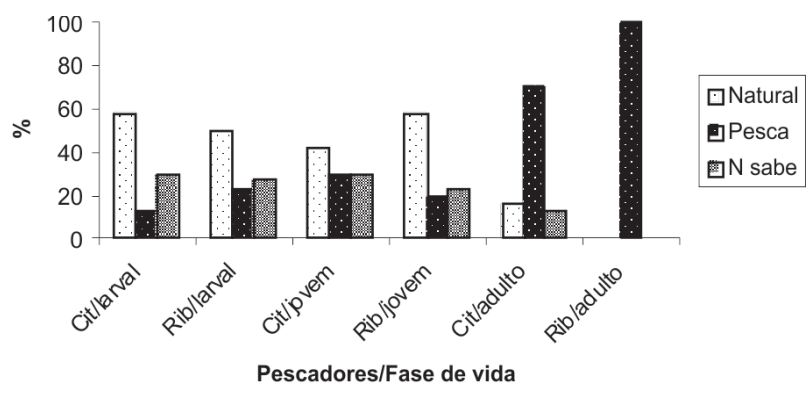

Figura 3 - Mortalidade do pirarucu nas fases larval, juvenil e adulta segundo os pescadores citadinos - Cit $(n=31)$ e Ribeirinhos - Rib $(n=26)$ da Amazônia Central. 


\section{CONCLUSÕES}

Os pescadores mostraram ter um conhecimento etnobiológico consistente sobre a ecologia e o comportamento do Pirarucu da regiáo. Essas informaçóes necessitam ser mais bem explorado para que pesquisas sobre co-manejo, conservação e uso sustentável dos recursos pesqueiros possam ser efetiva e eficazmente implementadas. Sendo assim, o presente estudo pretendeu contribuir com o desenvolvimento sustentável, verificando que os saberes locais dos pescadores em relação aos aspectos biológicos e ecológicos do pirarucu, podem ser incorporados como informaçóes úteis para implementação de futuros projetos de gestáo participativa no manejo pesqueiro da região.

\section{AGRADECIMENTOS}

Á Coordenação de Aperfeiçoamento de Pessoal de Nível Superior (CAPES) pelo financiamento da bolsa de pesquisa durante o mestrado, e ao Programa Integrado de Recursos Aquáticos da Várzea da Universidade Federal do Amazonas (PYRÁ-UFAM) pelo apoio logístico e institucional, e aos pescadores artesanais do porto desembarque de Manaus e aos moradores de Manacapuru pelas informaçóes concedidas nas entrevistas.

\section{BIBLIOGRAFIA CITADA}

Bard, J.; Imbiriba, E.P. 1986. Piscicultura do pirarucu, Arapaima gigas. Boletim Empresa Brasileira de Pesquisa Agropecuária Centro de Pesquisa Agropecuaria do Tropico Úmido, 52:17-20.

Batista, V.S. 1998. Distribuição, dinâmica da frota pesqueira e dos recursos pesqueiros da Amazônia Central. Tese de doutorado. Instituto Nacional de Pesquisa da Amazônia. 282pp.

Castello, L. 2004. A method to count pirarucu fishers, assessment and management. American Journal of Fisheries Management, 24:379-389.

Clark, J. R. 1996. Coastal zone management handbook. Florida (USA): CRC press. 694p.

Castello, L. 2008. Lateral migration of Arapaima gigas in floodplains on the Amazon. Ecology of Freshwater Fish, 17:38-46.

Costa Neto, E.M. 2001. A cultura pesqueira do litoral norte da Bahia: Etnoictiologia, desenvolvimento e sustentabilidade. Salvador: Ed EDUFBA; Maceió. 159 pp.

Crossa, M. Petrere, M. 1999. Morphmetric relationship and indirect determination of the length frequency structure of the pirarucu Arapaima gigas (Cuvier) in Brazilian Amazonian. Fisheries Management Ecology, 6: 233-240.

Ferreira, J.G.E.; Zuanon, J.A.S.; Santos, G.M. 1988. Peixes comerciais do médio amazonas: regiāo de Santarém, Pará. Ediçōes IBAMA, Brasília. 211 pp.

Fontenele, O. 1948. Contribuição para o conhecimento da biologia do pirarucu Arapaima gigas (Cuvier) em cativeiro (Actinopterigii, Osteoglossidae). Revista Brasileira de Biologia, 8: 445-459.
Fontenele, O.; Vasconcelos, E.A. 1982. O pirarucu, Arapaima gigas (Cuvier, 1817), nos açudes do nordeste: resultados de sua aclimataçáo e prováveis causas de depleção de seus estoques. Boletim Técnico DNOCS, 40: 43-66.

Furtado, L. 1993. Pescadores do rio Amazonas: Um estudo antropológico da pesca ribeirinha numa área amazônica. Coleção Eduardo Galvão. Belém. 486 pp.

Guerra, F. H. 1980. Desarrollo sexual Del paiche, Arapaima gigas, em las zonas reservadas del Estado (Rio Pacaya y Samira) 19711975. Informe do Iinstituto del Mar del Perú, 67:1-33.

Imbiriba, E.P. 2001. Potencial de criação de pirarucu, Arapaima gigas, em cativeiro. Acta Amazonica, 31: 299-316.

Imbiriba, E.P. 1994. Reprodução, larva e alevinagem do pirarucu (Arapaima gigas). Belém E Boletim - Recomendaçōes básicas. Empresa Brasileira de Pesquisa Agropecuária Centro de Pesquisa Agropecuaria do Tropico Umido,26:19.

Hrbek, T. Crossa, M. and Farias, I. P. 2007. Conservation Strategies for Arapaima gigas (Schinz, 1882) and The Amazonian Varzea ecosystem. Brazilian Journal Biological. 67: 909-917.

Isaac, V.J. Ruffino, M.L. 2000. Informe estatístico do desembarque pesqueiro na cidade de Santarém, PA: 1992 - 1993, p. 225-280. In: IBAMA. Recursos pesqueiros do médio Amazonas: biologia e estatística pesqueira, (Coleçáo Meio Ambiente. Serie Estudos Pesca, 22). Brasília.

Junk, N.J.; Honda, E. M. S. 1976. A pesca na Amazônia: aspectos ecológicos e econômicos, p. 211-226. In: Anais do I Encontro Nacional sobre Limnologia, Psicultura e Pesca Continental, Belo Horizonte: Fundação João Pinheiro.

Levine, D. M. Berenson, L. M. Stephan, D. 2000. Estatística teoria e aplicaçōes: usando o Microsoft ${ }^{\oplus}$ Excel em português. Editora JC. Rio de Janeiro. 819pp.

Lowe-Mcconnel, R.H. 1975. Fish communities in tropical freshwater: their distribution, Ecology and Evolution. Longmana. London. $382 \mathrm{pp}$.

Marques, J. G. W. 1991. Aspectos ecológicos na etnoictiologia dos pescadores do complexo estuarino-lagunar de Mandaú Manguaba, Alagoas. Tese de Doutorado, Instituto de Biolgoia, UNICAMP, Campinas (SP). 285pp.

Marques, J.G. W. 1995. Pescando pescadores: etnoecologia abrangente no baixo São Francisco. NUPAUB. São Paulo.250 pp.

Migdalski E.C. 1957. Contribution to the life history of the South American fish Arapaima gigas. Journal Copeia, 1: 54-56.

Murrieta, R. S. 2001. A mística do Pirarucu: pesca, ethos e paisagem em comunidades rurais no Baixo Amazonas. Horizontes Antropológicos, 16: 113-130.

Neves, A.M.B. 2000. Conhecimento atual sobre o pirarucu Arapaima gigas, p. 90-113. In: Fisher, C.F.A. (Coord). Recursos pesqueiros do médio Amazonas. Brasília: Ed. IBAMA.

Oliveira, V. Poleto, S.L. Venere, P.C. 2000. Feeding of juvenile pirarucu (Arapaima gigas, Arapaimidae) in their natural environment, Lago Quatro bocas, Araguaiana - MT, Brasil. Neotropical Ichthyology, 3: 312-314.2 
Petrere Jr, M, Barthem, R.B.; Magnawita, A. 1992. Relatório final do tema: utilizaçáo dos recursos hídricos minerais. Sub-tema: pesca e recursos pesqueiro, p.95-96. In: Seminário Internacional Sobre Meio Ambiente, Pobreza e Desenvolvimento da Amazônia. Sindamazônia. Belém.

Petrere Jr., M. 1992. As comunidades humanas ribeirinhas da Amazônia e suas transformaçóes sociais, p. 31-68. In: Diegues, A.C. (Ed) Populaçôes humanas, rios e mares da Amazônia. Anais do IV Encontro de Ciências Sociais e o Mar no Brasil, São Paulo.

Queiroz, H. L.; Sardinha, A.D. 1999. A preservaçáo e o uso sustentado do pirarucu de Mamirauá, 197 pp. In: Queiroz, H.L.; Cramptom, W. (Eds.) Estratégias para manejo de recursos pesqueiros em Mamirauá. CNPQ/ MCT. Brasília.

Queiroz, H.L. 2000. Natural history and conservation of pirarucu, Arapaima gigas at the Amazonian várzea: red grants in muddy water. Tese de Doutorado. University of St. Andrews, St. Andrews. 226 pp.

Ruddle, K. 2001. Systems of knowledge: dialogue, relationships and process. Environment, Development and Sustainability 2:277-304.
Santos, G.M.; Ferreira, E.G. 1999. Peixes da bacia amazônica, p. 345373. In: Lowe-McConnel, R.H. Estudos ecológicos de comunidades de peixes tropicais. Ed. EDUSP. São Paulo.

Seixas, C. S.; Begossi, A. 2000. Central place optmal foraging theory: population and individual analysis of fishing strategies at Aventureiro (Ilha Grande, Brazil). Ciência e Cultura 52 (2): $85-62$.

Souza, R.H. S. Val, A. L. 1991. O gigante das águas doces. Ciência Hoje. 64: 129-33.

ZAR, J.H. 1999. Biostatistical Analysis. New Jersey. Ed. PrenticeHall. 663pp.

Recebido em: 11/02/2011

Aceito em: 30/11/2011 
